\title{
Athermal Holographic Filters
}

\author{
Hung-Te Hsieh, George Panotopoulos, Matthieu Liger, Yu-Chong Tai, Member, IEEE, and \\ Demetri Psaltis, Senior Member, IEEE
}

\begin{abstract}
This letter presents the theory and experimental results of an athermal holographic filter design employing a thermally actuated microelectromechanical system mirror to compensate for the drift of Bragg wavelength due to changes of temperature. The center wavelength of our holographic filter is shown to remain constant from $21^{\circ} \mathrm{C}$ to $60^{\circ} \mathrm{C}$.
\end{abstract}

Index Terms-Gratings, holographic filters, temperature compensations, wavelength-division multiplexing (WDM).

\section{INTRODUCTION}

$\mathbf{H}$ OLOGRAPHIC filters [1] have been used for wavelength-division-multiplexing (WDM) filtering applications [2], [3]. Temperature dependence is a critical concern for telecommunications. In thin film filter technology, proper selection of materials can combat the thermal drift of Bragg wavelengths [4]. Athermal filter designs were also reported for arrayed-waveguide gratings [5] and fiber Bragg gratings [6]. However, such flexibility is not available in bulk holographic filters. In this letter, a feasible system solution is proposed and experimentally shown to compensate for the effects of temperature change.

\section{THEORY}

A grating holographically imprinted inside a recording material can be operated as a WDM filter in the reflection geometry, as shown in Fig. 1.

The wavelength satisfying the grating equation (1) will be strongly reflected, whereas the other wavelengths pass through the filter unaffected

$$
\lambda_{B}=2 n\left(T_{0}\right) \Lambda\left(T_{0}\right) \cos \theta_{B}
$$

where $n\left(T_{0}\right)$ is the refractive index of the material at $\lambda_{B}$ at temperature $T_{0}$ and $\Lambda\left(T_{0}\right)$ is the period of the index grating at $T_{0}$. By inspecting (1), we notice that we can Bragg match the grating to a shorter wavelength if we tilt the incident beam away from the normal.

Temperature changes affect holographic filters mainly through two mechanisms. (Other possible effects will be neglected here, e.g., the thermal dependence of the piezoelectric tensor will manifest itself when stress is being applied.)

1) Thermal expansion or contraction of the bulk material (in our experiments, $\mathrm{LiNbO}_{3}: \mathrm{Fe}$ ).

Manuscript received June 2, 2003; revised August 15, 2003. This work was supported by the Engineering Research Centers Program of the National Science Foundation under Award EEC-9402726.

The authors are with the Department of Electrical Engineering, California Institute of Technology, Pasadena, CA 91125 USA (e-mail: tete@caltech.edu; psaltis@ caltech.edu).

Digital Object Identifier 10.1109/LPT.2003.820483

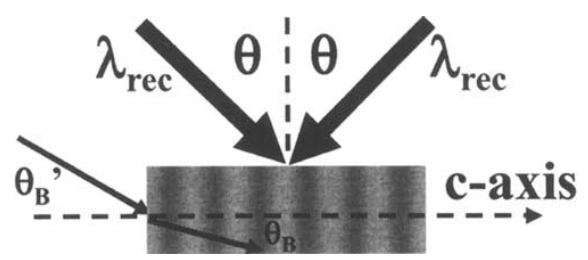

Fig. 1. Recording a holographic grating inside a $\mathrm{LiNbO}_{3}$ crystal at $\lambda_{\text {rec }}=$ $488 \mathrm{~nm}$ in the transmission geometry and then operating it as a WDM filter in the reflection geometry.

2) Thermal dependence of the dielectric constant of the bulk material.

Assume the Bragg wavelength of the filter is $\lambda_{B}$ corresponding to an incident angle $\theta_{B}\left(\theta_{B}\right.$ is the angle measured inside the crystal, whereas $\theta_{B}{ }^{\prime}$ is measured outside the crystal) at temperature $T_{0}$. When the temperature changes to $T_{0}+\Delta T$, the Bragg wavelength of the filter will have a corresponding shift and move to $\lambda_{B}+\Delta \lambda$. If we adjust the incident angle by $\Delta \theta$ such that the Bragg wavelength shifts back to $\lambda_{B}$, we will have

$$
\lambda_{B}=2 n\left(T_{0}+\Delta T\right) \Lambda\left(T_{0}+\Delta T\right) \cos \left(\theta_{B}+\Delta \theta\right)
$$

If the thermal expansion coefficient (TEC) and the refractive index dependence on temperature are both linear within the temperature range of interest, we have $n\left(T_{0}+\Delta T\right) \cong n\left(T_{0}\right)(1+$ $a \Delta T)$ and $\Lambda\left(T_{0}+\Delta T\right) \cong \Lambda\left(T_{0}\right)(1+b \Delta T)$. From (1) and (2), we can derive a relation between $\Delta \theta$ and $\Delta T$ for a constant Bragg wavelength in spite of temperature changes

$$
\frac{\cos \left(\theta_{B}+\Delta \theta\right)}{\cos \theta_{B}}=\frac{1}{(1+a \Delta T)(1+b \Delta T)} .
$$

Since both thermal coefficients $a$ and $b$ are positive [7], [8], we conclude that as the temperature rises, the Bragg wavelength of a given filter will shift upward, i.e., to a longer wavelength. To compensate for such a shift, we tilt the beam away from the normal. On the other hand, to undo the effect caused by a temperature drop, we adjust the beam toward the normal.

Based on (3), we propose an athermal design to maintain the Bragg wavelengths of WDM filters invariant with respect to temperature fluctuations. The principle of operation is illustrated in Fig. 2. We use a bimetallic composite beam to control the direction of the incident light beam. The device makes use of the TEC discrepancy between two properly chosen materials (in our case, aluminum and silicon) and deflects as the temperature changes [9]. We operate the filter away from normal incidence because compensation for negative temperature drifts cannot be carried out near normal incidence and to avoid nonlinearities ( $\Delta \theta$ is a highly nonlinear function of $\Delta T$ near normal incidence). 


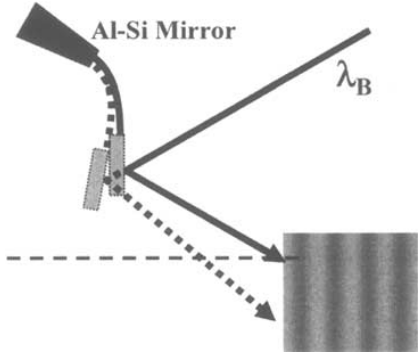

Fig. 2. Athermal design of holographic filter utilizing an Al-Si composite beam microactuator whose tip deflects as the temperature changes.

\section{EXPERIMENT AND RESULTS}

In our experiments, holographic filters are recorded in an iron-doped lithium niobate $\left(\mathrm{LiNbO}_{3}: \mathrm{Fe}, 0.05\right.$ wt $\left.\% \mathrm{Fe}_{2} \mathrm{O}_{3}\right)$ crystal by interfering two coherent continuous-wave laser beams inside the crystal, as shown in Fig. 1. The crystal sample dimensions are $2.0 \times 1.0 \times 0.3 \mathrm{~cm}$ with its $c$-axis running parallel to the longest edge and the concentration of $\mathrm{Fe}^{2+}$ is $1.283 \times 10^{24} \mathrm{~m}^{-3}$. A stabilization system has been incorporated into the recording setup in order to prevent the interference pattern from drifting. By properly choosing the angle $2 \theta$ between the recording beams, we are able to control the Bragg wavelength of the grating operated in the reflection geometry. The transmittance of a typical filter at an incident angle $\theta_{B}^{\prime}=5^{\circ}$ is shown in Fig. 3(a) for three different temperatures. The Bragg wavelength $\lambda_{B}$ of the recorded filter at $21.79^{\circ} \mathrm{C}$ is measured to be $1556.61 \mathrm{~nm}$. The filter efficiency is dropping due to hologram decay at elevated temperatures since the hologram was not thermally fixed [10].

To specify the microelectromechanical systems (MEMS) mirror parameters, we first figure out the Bragg wavelengths for a series of incident angles at four different temperatures $\left(21.79{ }^{\circ} \mathrm{C}, \quad 33.36{ }^{\circ} \mathrm{C}, 45.68{ }^{\circ} \mathrm{C}, 58.46{ }^{\circ} \mathrm{C}\right)$. Temperature monitoring is made possible by reading the resistance off a thermistor in close contact with the $\mathrm{LiNbO}_{3}$ crystal when the whole system is in thermal equilibrium. A thermoelectric (TE) cooler is used to control the temperature of the system. The Bragg wavelength corresponding to the incident angle $\theta_{B}^{\prime}=5^{\circ}\left(\theta_{B} \cong 2.25^{\circ}\right)$ at the lowest temperature is chosen as the target wavelength that will be maintained constant through angular compensation. By doing a fit of the data to (3), we end up with the optimal compensation angles as a function of temperature change. This is plotted as a solid line in Fig. 4.

Our data suggest that for operation around an incident angle $\theta_{B}^{\prime}=5^{\circ}$, an angular correction of $1.18^{\circ}$ will be required for a temperature change of $100{ }^{\circ} \mathrm{C}$. The aluminum-silicon composite beam was designed to deflect about $0.59^{\circ}$ for a temperature change of $100^{\circ} \mathrm{C}$.

A photograph of the MEMS mirror is shown in Fig. 5. It is composed of two parts: a mirror part (surface dimensions $1.0 \times$ $3.0 \mathrm{~mm}, 50 \mathrm{~nm}$ of gold deposited on $0.45-\mathrm{mm}$ silicon gives a reflectance of $85 \%$ at $1550 \mathrm{~nm}$ ) and a beam (surface dimensions $2.0 \times 0.5 \mathrm{~mm}, 500-\mathrm{nm}$ aluminum deposited on $0.025-\mathrm{mm}$ silicon), which deflects as the temperature varies. The measured deflection angle of the mirror as a function of temperature is also plotted in Fig. 4.

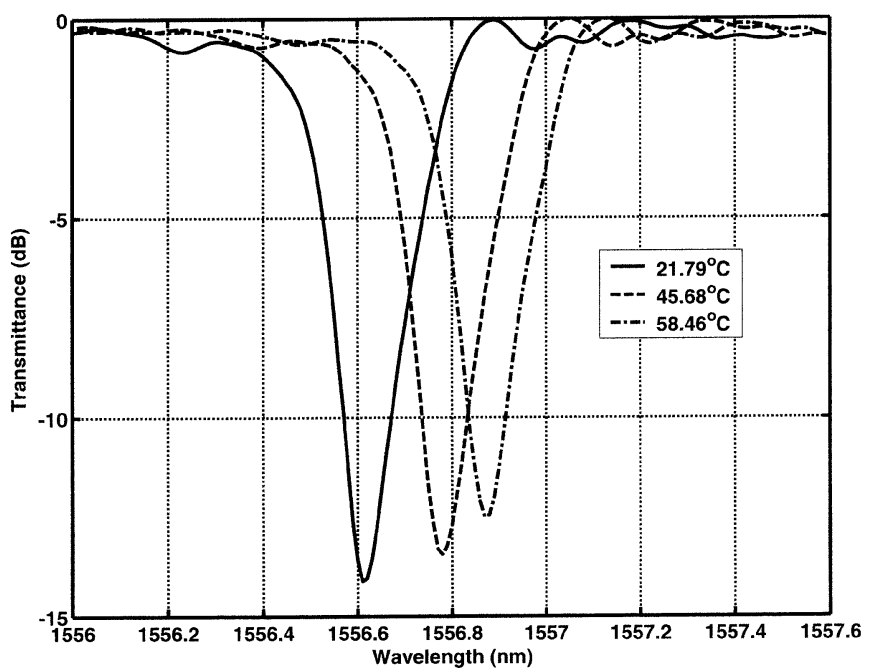

(a)

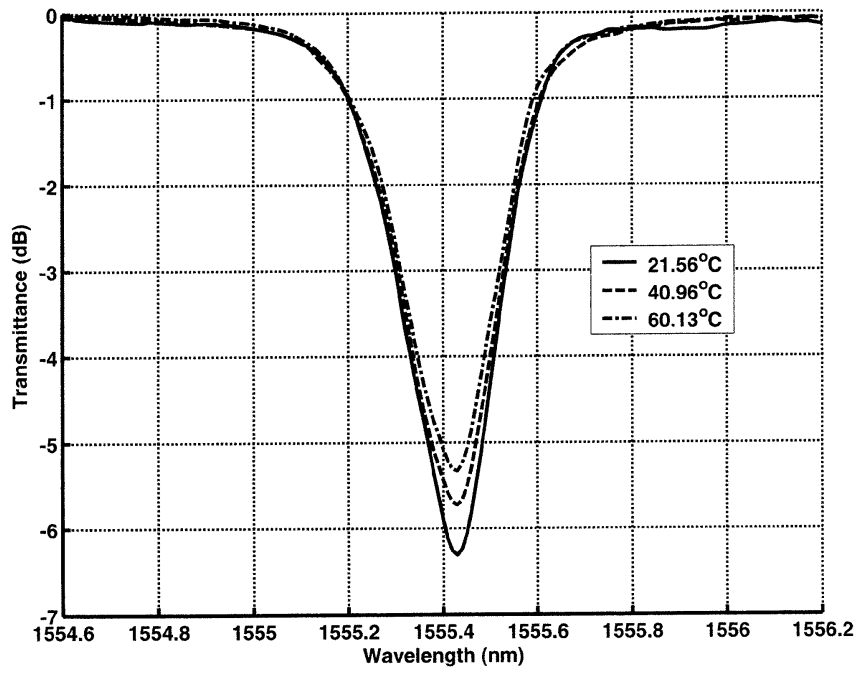

(b)

Fig. 3. (a) Filter response measured in the through channel at $\theta_{B}^{\prime}=5^{\circ}$ for three different temperatures. (b) Filter response measured in the through channel at $\theta_{B}^{\prime}=5^{\circ}$ for three different temperatures with the compensating MEMS mirror.

We mount the holographic filter and the MEMS mirror on two separate TE coolers as depicted in Fig. 5. Two identical thermistors are used to monitor the temperatures of the filter and the mirror. The output from a tunable laser is reflected off the mirror toward the filter at an (outside) incident angle of $5^{\circ}$. At this point both the filter and the mirror are at room temperature. The filter response is measured and the Bragg wavelength is determined. Then the TE coolers are turned on and raise the temperatures of both. The readings of the two thermistors are kept the same throughout the measurements of filter response. The filter shapes at $\theta_{B}^{\prime}=5^{\circ}$ for three different temperatures are plotted in Fig. 3(b). Compared with Fig. 3(a), the drift of the Bragg wavelength is indeed compensated for by the deflection of the mirror. The compensation can be done easily in practice because the response time of the MEMS mirror is much shorter than that of the bulkier $\mathrm{LiNbO}_{3}$ crystal.

In Fig. 6, we plot the Bragg wavelengths measured with the athermal design for three different incident angles. The dashed 


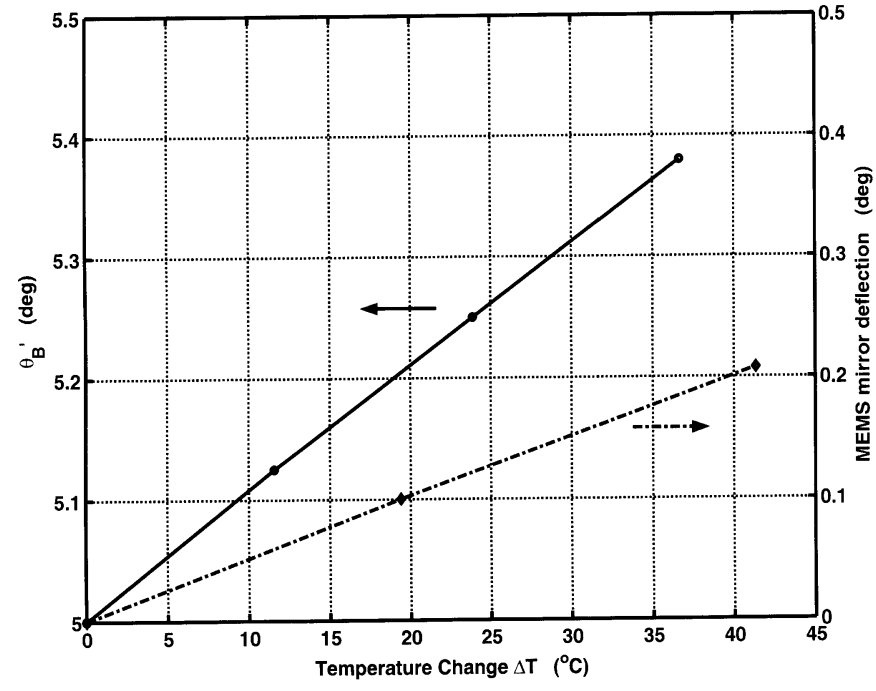

Fig. 4. Solid curve represents the calculated optimal compensation angle $\theta_{B}^{\prime}$ as a function of temperature change $\Delta T$. The dashed-dotted curve is the measured angular deflection of the MEMS mirror subject to $\Delta T$.

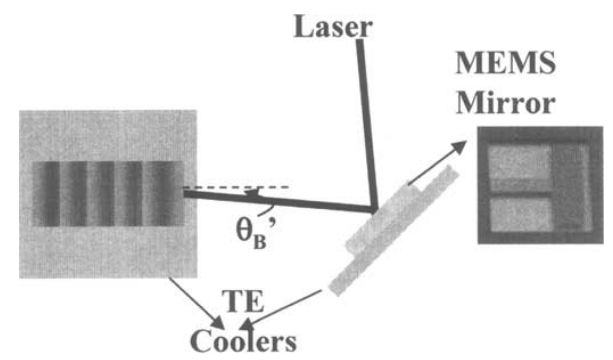

Fig. 5. Experimental setup combining the thermally driven MEMS mirror with the recorded holographic filter to realize the athermal filter design. A picture of the MEMS mirror is also shown.

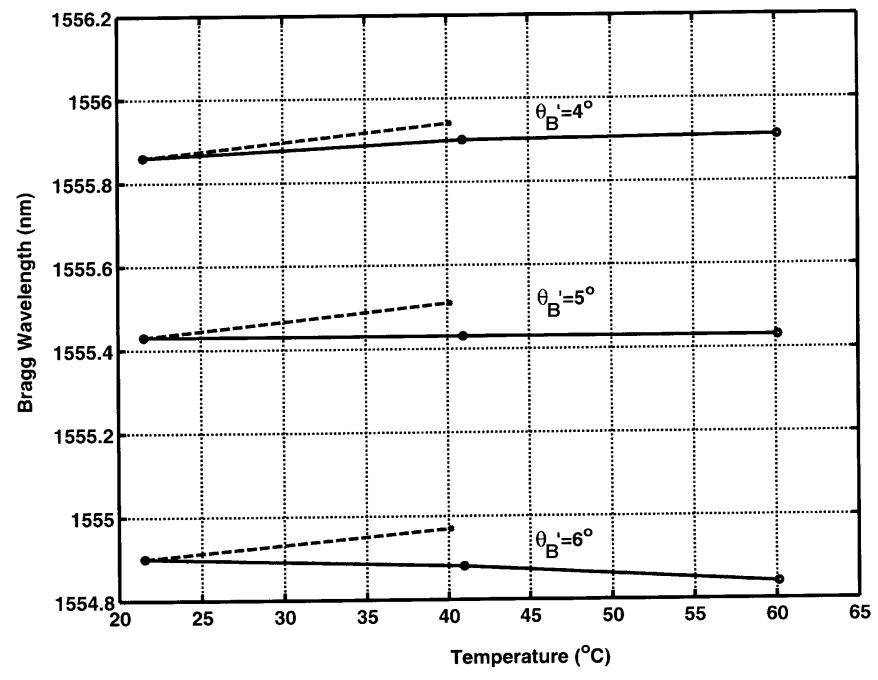

Fig. 6. Bragg wavelengths measured with the athermal design for three different incident angles versus temperature.

lines indicate the drifts of Bragg wavelengths due to the raised temperature in the absence of mirror compensation. We can see that for $\theta_{B}^{\prime}=5^{\circ}$, the Bragg wavelength remains constant between $21^{\circ} \mathrm{C}$ and $60^{\circ} \mathrm{C}$, which corroborates the validity of our athermal design. Without the athermal mechanism, the Bragg wavelength would drift about $0.16 \mathrm{~nm}(20 \mathrm{GHz})$ from $21^{\circ} \mathrm{C}$ to $60^{\circ} \mathrm{C}$. The Bragg wavelengths for $\theta_{B}^{\prime}=4^{\circ}$ are seen to increase with the rising temperature despite the counteracting MEMS operation although they do not increase quite as much as when there is no compensation mechanism; on the other hand, the Bragg wavelengths for $\theta_{B}^{\prime}=6^{\circ}$ are decreasing with the rising temperature because they are overcompensated by the MEMS mirror deflection. The athermal WDM filter design employing the MEMS mirror keeps the Bragg wavelength constant; however, to couple the reflected or transmitted beam back into a fiber, another counteracting MEMS mirror must be used to undo the beam walkoff.

Since many thermally actuated MEMS devices consist of materials that possess different mechanical properties, they can suffer from hysteresis. The Bragg wavelength of the athermal filter system undergoes a $0.09-\mathrm{nm}(11.25 \mathrm{GHz})$ decrease after we cool it back down to room temperature. A possible solution to this problem is to anneal the MEMS device at an elevated temperature (about $300{ }^{\circ} \mathrm{C}$ ) before use. The drift in the Bragg wavelength due to hysteresis was experimentally shown to reduce to $0.01 \mathrm{~nm}(1.25 \mathrm{GHz})$ after annealing.

\section{CONCLUSION}

We have shown that the temperature dependence of the Bragg wavelength of a holographic filter can be compensated by incorporating a passive thermally actuated MEMS mirror into the system. The packaging should be such that the mirror and the filter thermally track each other. To improve the performance of the athermal filter design, it is important to gain a deeper understanding of the evolution of filter shapes away from normal incidence. Other topics such as beam walkoff, polarization dependent loss, and the elimination of mirror hysteresis are also important considerations in practical applications.

\section{REFERENCES}

[1] G. A. Rakuljic and V. Leyva, "Volume holographic narrow-band optical filter," Opt. Lett., vol. 18, pp. 459-461, Mar. 1993.

[2] S. Breer and K. Buse, "Wavelength demultiplexing with volume phase holograms in photorefractive lithium niobate," Appl. Phys. B, vol. 66, pp. 339-345, Mar. 1998.

[3] D. Psaltis, "Coherent optical information systems," Science, vol. 298, pp. 1359-1363, Nov. 2002.

[4] H. Takashashi, "Temperature stability of thin-film narrow-bandpass filters produced by ion-assisted deposition," Appl. Opt., vol. 34, pp. 667-675, Feb. 1995.

[5] N. Keil, H. H. Yao, and C. Zawadzki, "Athermal polarization-independent arrayed-waveguide grating (AWG) multiplexer using an all-polymer approach," Appl. Phys. B, vol. 73, pp. 619-622, Nov. 2001.

[6] Y. L. Lo and C. P. Kuo, "Packaging a fiber Bragg grating without preloading in a simple athermal bimaterial device," IEEE Trans. Adv. Packag., vol. 25, pp. 50-53, Feb. 2002.

[7] R. T. Smith and F. S. Welsh, "Temperature dependence of elastic, piezoelectric, and dielectric constants of lithium tantalite and lithium niobate," J. Appl. Opt., vol. 42, pp. 2219-2230, May 1971.

[8] U. Schlarb and K. Betzler, "Refractive indices of lithium niobate as a function of temperature, wavelength, and composition-a generalized fit," Phys. Rev. B, vol. 48, pp. 15 613-15 620, Dec. 1993.

[9] W. H. Chu, M. Mehregany, and R. L. Mullen, "Analysis of tip deflection and force of a bimetallic cantilever microactuator," J. Micromech. Microeng., vol. 3, pp. 4-7, Feb. 1993.

[10] D. L. Staebler and J. J. Amodei, "Thermally fixed holograms in $\mathrm{LiNbO}_{3}$," Ferroelectrics, vol. 3, pp. 107-113, 1972. 